\title{
QUINOLIN-6-AMINES: SYNTHESIS AND BIOLOGICAL EVALUATION
}

\author{
Hament Panwar ${ }^{1, *}$, Nidhi Chaudhary ${ }^{2}$, Ranjana Dubey ${ }^{3}$, and Tilak Ram ${ }^{4}$ \\ ${ }^{1}$ Department of Chemistry, Neelkanth Institute of Technology, Modipuram-250110, Meerut, U.P., India \\ ${ }^{2}$ Department of Chemistry, M.I.E.T., Meerut-250001, U.P., India \\ ${ }^{3}$ Department of Chemistry, S.R.M. University, Modinagar-201204, Ghaziabad, U.P., India \\ ${ }^{4}$ Department of Chemistry, Govt. P.G. College, Uttarkashi-249193, U.K., India
}

Received July 12, 2013; Accepted August 22, 2013

\begin{abstract}
Some novel 2-\{(chloromethoxy)methyl\}thio-N-substituted phenyl-[1,2,4]triazolo[1,5-a] quinolin-6-amines have been derived from 5-bromocoumarin. All the synthesized compounds have been characterized by elemental and spectral (I.R., ${ }^{1} \mathrm{H}-\mathrm{NMR}$, Mass) analysis and evaluated for their antimicrobial, insecticidal and anthelmintic activities.
\end{abstract}

Keywords: Quinolin-6-amines; antimicrobial; insecticidal; anthelmintic

\section{ABSTRAK}

Beberapa senyawa baru kuinolin-6-amin tersubstitusi 2-\{(klorometoksi)metil\}tio- $N$ telah dihasilkan sebagai turunan dari 5-bromocoumarin. Semua senyawa yang disintesis telah dikarakterisasi melalui analsis unsur dan spektra (IR, H-NMR dan massa) dan diuji terhadap aktivitas antimikrobia, insentisida dan antelmintak.

Kata Kunci: kuinolin-6-amin; antimikrobia; insektisida; antelmintak

\section{INTRODUCTION}

Coumarins belong to a group of compounds known as the benzopyrones, all of which consist of a benzene ring joined to a pyranone ring. During 1820, coumarin [1] was first isolated from the tonka bean and the name coumarin derives from a French word, coumarou, for the tonka bean. Due to its sweet odor, it was frequently used in perfumes since 1882. Coumarin and its derivatives extensively used as building block in heterocyclic synthetic chemistry for their versatile biological activities including antibacterial [2-5] antifungal [6-10], herbicidal [11], antitumor [12-13], anti-HIV [14], anticoagulant [15], anticonvulsant [16-17], anti-inflammatory [18-19], antimalarial [20] activities. In addition, these compounds are used as additives in food and cosmetics [21], dyes for tuning lasers [22-25]. On the other side, 1,2,4triazoles symbolize one of the most biologically active block of compounds possessing a wide spectrum of activities. The 1,2,4-triazole derivatives were allied with various pharmacological activities viz. antiseptic, analgesic, anti-convulsant [26-37], antibiotic [26], antiallergic [26], anti-inflammatory [26-35,38], diuretic $[26,30,33]$, fungicidal [28-29,35-38], insecticidal $[28,35,38]$, herbicidal $[28,35,38]$, antibacterial $[28-31,36-$ $37]$, antiviral [27-30,32-33,35], antidepressant [27,30,34], antimicrobial [27-30,32,35-37], antitumor [28,31,34-35], antihypertensive $[30,33,34]$, and antimigraine

* Corresponding author. Tel/Fax : +91-121-2578204

Email address : dr_h.panwar@rediffmail.com compounds [32]. There are several well known market drugs which possess 1,2,4-triazole group e.g. rizatriptan, nefazodone, vorozole, ribavirin, fluconazole, letrozole and uniconazole etc. These above mentioned observations motivated us to design a synthetic strategy (Scheme-1). Herein we report on the synthesis of a new class of substituted quinolin-6-amines and their biological activity by using a simple synthetic approach from easily available building block materials.

\section{EXPERIMENTAL SECTION}

\section{Materials}

All the chemicals used for the preparation of desired derivatives, were obtained from Sisco Research Laboratories (SRL), Mumbai, India; Qualigen Fine Chemicals, Mumbai, India; E. Merck Ltd., New Delhi, India. The reference drugs ampicillin trihydrate, fluconazole, cypermethrin and albendazole were procured from Ind-Swift Pharmaceutical, Panjab; Cadilla Pharmaceuticals, Gujarat; Royal Crop Science, Panipat, India.

\section{Instrumentation}

The melting points of the compounds were determined in open glass capillaries with the help of 


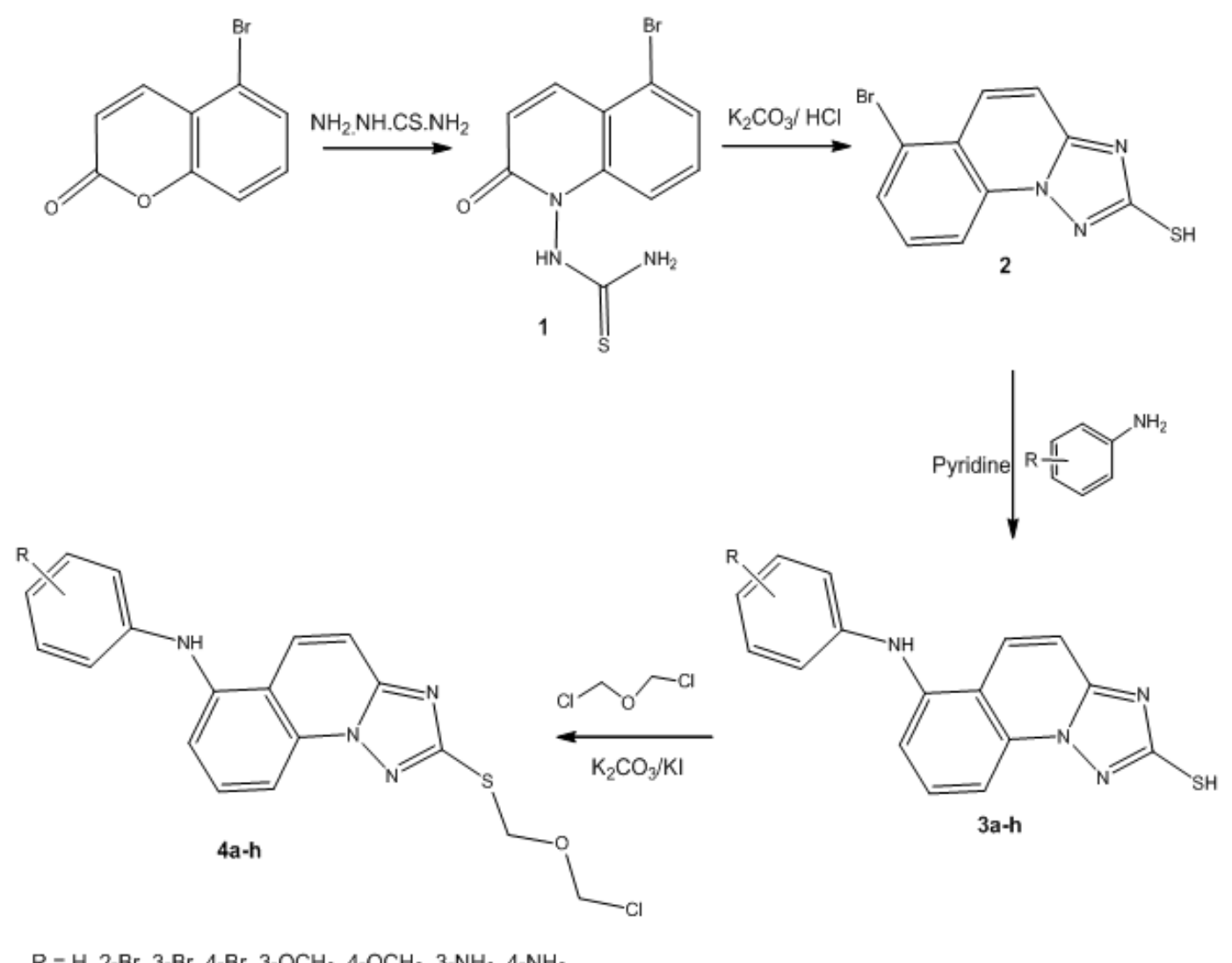

Scheme 1

thermonic melting points apparatus (Campbell Electronics, Mumbai, India) and are uncorrected. The homogeneity of all the newly synthesized compounds was routinely checked by TLC on silica gel G plates and spots were located by using iodine chamber. Elemental analysis was performed in Heraeus $\mathrm{CHN}$ rapid analyzer. The results were found within the $\pm 0.4 \%$ of theoretical values. Infrared spectra were recorded on $\mathrm{KBr}$ pellets on a Perkin Elmer system 2000 FTIR spectrometer and ${ }^{1} \mathrm{H}$-NMR spectra on Bruker DPX 200 using TMS as internal standard.

\section{Characterization of the Synthesized Compounds}

The formation of compound 1 was confirmed by the presence of absorption bands at 1625, 1350, 3400, 3150 $\mathrm{cm}^{-1}$ due to vibrational movement of $\mathrm{C}=\mathrm{O}, \mathrm{C}=\mathrm{S}, \mathrm{NH}_{2}, \mathrm{NH}$ of incorporated thiourea group respectively which was further evidenced by the appearance of broad signals in ${ }^{1} \mathrm{H}-\mathrm{NMR}$ spectrum at $\delta 6.58$ and 4.30 due to $-\mathrm{NH}$ and $-\mathrm{NH}_{2}$ groups. Disappearance of adsorption band for $\mathrm{C}-\mathrm{O}-\mathrm{C}$ at $1040 \mathrm{~cm}^{-1}$ clears the formation of compound 1. Cyclisation of compound 1 into compound 2 was confirmed by the disappearance of vibration band in I.R. spectrum at $3400 \mathrm{~cm}^{-1}$ of $\mathrm{NH}_{2}$ group and appearance of bands at 2400, $1625 \mathrm{~cm}^{-1}$ for $-\mathrm{SH}$ and $\mathrm{C}=\mathrm{N}$ groups respectively. ${ }^{1} \mathrm{H}-\mathrm{NMR}$ spectra revealed the anilation of compound 2 to compounds $3 \mathrm{a}-\mathrm{h}$; by the appearance of broad signal at $\delta 3.70$. Presence of band at $1025 \mathrm{~cm}^{-1}$ is the characteristic feature of the existence of compounds $4 a-h$ which was further evidenced by the disappearance of band for $\mathrm{SH}$ at $2400 \mathrm{~cm}^{-1}$.

\section{Procedure}

\section{Synthesis of 1-(2-oxoquinolin-1(2H)-yl)thiourea 1}

A reaction mixture of 5 -bromo coumarin $(0.01$ $\mathrm{mol})$ and thiosemicarbazide $(0.01 \mathrm{~mol})$ in pyridine was refluxed for $3 \mathrm{~h}$. The progress of reaction was monitored by TLC. Excess of the solvents was removed under reduced pressure. Residue was washed with water, filtered, dried, triturated with petroleum ether $\left(40-60{ }^{\circ} \mathrm{C}\right)$ to yield compound 1: Yield $83 \%$, m.p. $89^{\circ} \mathrm{C}$. IR (KBr) $v_{\max }$ in $\mathrm{cm}^{-1}: 1290(\mathrm{~N}-\mathrm{N}), 1350$ $(\mathrm{C}=\mathrm{S}), 1530$ (C-C of aromatic), $1615(\mathrm{C}=\mathrm{N}), 1640$ $(\mathrm{C}=\mathrm{O}), 3030$ (aromatic $\mathrm{CH}), 3180(\mathrm{NH}), 3400\left(\mathrm{NH}_{2}\right)$. H-NMR $\left(\mathrm{CDCl}_{3}\right) \delta: 4.30$ (bs, $\left.2 \mathrm{H}, \mathrm{NH}_{2}\right), 6.15(\mathrm{~s}, 1 \mathrm{H}$, $\mathrm{NH})$, 6.85-7.87 (m, 6H, Ar-H). MS: m/z $298.96[\mathrm{M}]^{+}$. Elemental analysis $\left(\mathrm{C}_{10} \mathrm{H}_{8} \mathrm{~N}_{3} \mathrm{SOBr}\right)$, calcd: $\mathrm{C} 40.28$, H 2.70, N 14.09\%, found: C 40.16, H 2.73, N 14.05\%. 


\section{Synthesis of 6-bromo-(1,2,4-triazolo)(1,5-a) quinoline -2-thiol 2}

Compound $1(0.01 \mathrm{~mol})$ was mixed with aqueous solution of $\mathrm{K}_{2} \mathrm{CO}_{3}(25 \% \mathrm{w} / \mathrm{w})$ and stirred at $55^{\circ} \mathrm{C}$ for $8 \mathrm{~h}$. On completion, the reaction mixture cooled and acidified with $2 \% \mathrm{HCl}$. The crude solid washed with water, filtered, dried and recrystallized with dioxane to obtain compound 2: Yield $76 \%$, m.p. $137{ }^{\circ} \mathrm{C}$. IR $(\mathrm{KBr}) \mathrm{V}_{\max }$ in $\mathrm{cm}^{-1}$ : $1288(\mathrm{~N}-\mathrm{N}), 1533$ (C-C of aromatic), $1610(\mathrm{C}=\mathrm{N}), 3035$ (aromatic $\mathrm{CH}) .{ }^{1} \mathrm{H}-\mathrm{NMR}\left(\mathrm{CDCl}_{3}\right)$ $\delta: 6.67-7.56(\mathrm{~m}, 5 \mathrm{H}$, $\mathrm{Ar}-\mathrm{H}), 12.70$ (s, 1H, HS). MS: m/z $280.94 \quad[\mathrm{M}]^{+}$. Elemental analysis $\left(\mathrm{C}_{10} \mathrm{H}_{6} \mathrm{~N}_{3} \mathrm{SBr}\right)$, calcd: $\mathrm{C} 42.87$, H 2.16, N 15.00\%, found: C 42.90, H 2.15, N 15.03\%.

\section{Synthesis of 6-(substitutedanilino)(1,2,4-triazolo) (1,5-a)quinoline-2-thiol 3a-h}

Methanolic solution of compound $2(0.01 \mathrm{~mol})$ in presence of few drops of pyridine was refluxed with different substituted anilines $(0.01 \mathrm{~mol})$ for $5-8 \mathrm{~h}$. On completion of the reaction, excess of solvent was distilled off and thus obtained residue cooled, poured into ice cold water, filtered, dried and triturated with petroleum ether $\left(40-60{ }^{\circ} \mathrm{C}\right)$ and recrystallized with appropriate solvents to furnish the products $3 a-h$.

6-(Anilino)(1,2,4-triazolo)(1,5-a)quinoline-2-thiol 3a. Yield $76 \%$, m.p. $137^{\circ} \mathrm{C}$. IR ( $\left.\mathrm{KBr}\right) \mathrm{v}_{\max }$ in $\mathrm{cm}^{-1}: 1288(\mathrm{~N}-\mathrm{N})$, 1533 (C-C of aromatic), $1610(\mathrm{C}=\mathrm{N}), 3035$ (aromatic $\mathrm{CH}), 3385(\mathrm{NH}) .{ }^{1} \mathrm{H}-\mathrm{NMR}\left(\mathrm{CDCl}_{3}\right)$ ס: 5.85 (bs, 1H, NH), 6.79-7.64 (m, 10H, Ar-H), 12.55 (s, 1H, HS). MS: m/z $292.08[\mathrm{M}]^{+}$. Elemental analysis $\left(\mathrm{C}_{16} \mathrm{H}_{12} \mathrm{~N}_{4} \mathrm{~S}\right)$, calcd: $\mathrm{C}$ 65.73, H 4.14, N 19.16\%, found: C 65.70, H 4.17, N $19.09 \%$.

6-(2-Bromoanilino)(1,2,4-triazolo)(1,5-a)quinoline-2thiol 3b. Yield $68 \%$, m.p. $169{ }^{\circ} \mathrm{C}$. IR (KBr) $\mathrm{v}_{\max }$ in $\mathrm{cm}^{-1}$ : $1290(\mathrm{~N}-\mathrm{N}), 1531$ (C-C of aromatic), $1615(\mathrm{C}=\mathrm{N})$, 3030 (aromatic $\mathrm{CH}), 3377(\mathrm{NH}) .{ }^{1} \mathrm{H}-\mathrm{NMR}\left(\mathrm{CDCl}_{3}\right) \delta: 5.77$ (bs, 1H, NH), 6.68-7.60 (m, 9H, Ar-H), 12.41 (s, 1H, HS). MS: $\mathrm{m} / \mathrm{z} 369.99[\mathrm{M}]^{+}$. Elemental analysis $\left(\mathrm{C}_{16} \mathrm{H}_{11} \mathrm{~N}_{4} \mathrm{~S} \mathrm{Br}\right)$, calcd: C 51.76, H 2.99, N 15.09\%, found: C 51.70, H 2.97, N 15.14\%.

6-(3-Bromoanilino)(1,2,4-triazolo)(1,5-a)quinoline-2thiol 3c. Yield $63 \%$, m.p. $158{ }^{\circ} \mathrm{C}$. IR $(\mathrm{KBr}) \mathrm{v}_{\max }$ in $\mathrm{cm}^{-1}$ : $1292(\mathrm{~N}-\mathrm{N}), 1529$ (C-C of aromatic), $1611(\mathrm{C}=\mathrm{N})$, 3032 (aromatic $\mathrm{CH}), 3380(\mathrm{NH}) .{ }^{1} \mathrm{H}-\mathrm{NMR}\left(\mathrm{CDCl}_{3}\right) \delta: 5.80$ (bs, 1H, NH), 6.65-7.55 (m, 9H, Ar-H), 12.46 (s, 1H, HS). MS: $\mathrm{m} / \mathrm{z} 369.99[\mathrm{M}]^{+}$. Elemental analysis $\left(\mathrm{C}_{16} \mathrm{H}_{11} \mathrm{~N}_{4} \mathrm{~S} \mathrm{Br}\right)$, calcd: C 51.76, H 2.99, N 15.09\%, found: C 51.70, H 2.97, N 15.14\%.

6-(4-Bromoanilino)(1,2,4-triazolo)(1,5-a)quinoline-2thiol 3d. Yield $70 \%$, m.p. $162{ }^{\circ} \mathrm{C}$. IR $(\mathrm{KBr}) \mathrm{v}_{\max }$ in $\mathrm{cm}^{-1}$ : $1283(\mathrm{~N}-\mathrm{N}), 1526$ (C-C of aromatic), $1617(\mathrm{C}=\mathrm{N})$, 3024 (aromatic $\mathrm{CH}), 3373(\mathrm{NH}) .{ }^{1} \mathrm{H}-\mathrm{NMR}\left(\mathrm{CDCl}_{3}\right)$ ס: 5.72 (bs, 1H, NH), 6.60-7.61 (m, 9H, Ar-H), 12.38 (s, 1H, HS). MS: $\mathrm{m} / \mathrm{z} 369.99[\mathrm{M}]^{+}$. Elemental analysis $\left(\mathrm{C}_{16} \mathrm{H}_{11} \mathrm{~N}_{4} \mathrm{~S} \mathrm{Br}\right)$, calcd: C 51.76, H 2.99, N 15.09\%, found: C 51.70, H 2.97, N 15.14\%.

6-(3-Methoxyanilino)(1,2,4-triazolo)(1,5-a)quinoline2-thiol 3e. Yield $66 \%$, m.p. $135^{\circ} \mathrm{C}$. IR $(\mathrm{KBr}) \mathrm{v}_{\max }$ in $\mathrm{cm}^{-1}$ : $1279(\mathrm{~N}-\mathrm{N}), 1527(\mathrm{C}-\mathrm{C}$ of aromatic), $1614(\mathrm{C}=\mathrm{N})$, 3028 (aromatic $\mathrm{CH}), 3376(\mathrm{NH}),{ }^{1} \mathrm{H}-\mathrm{NMR}\left(\mathrm{CDCl}_{3}\right)$ ס: 3.81 (s, 3H, $\left.\mathrm{OCH}_{3}\right), 5.76$ (bs, $\left.1 \mathrm{H}, \mathrm{NH}\right), 6.58-7.55(\mathrm{~m}$, $9 \mathrm{H}, \mathrm{Ar}-\mathrm{H}$ ), 12.44 (s, 1H, HS). MS: m/z $322.09[\mathrm{M}]^{+}$. Elemental analysis $\left(\mathrm{C}_{17} \mathrm{H}_{14} \mathrm{~N}_{4} \mathrm{SO}\right)$, calcd: C 63.33, H 4.38, N 17.38\%, found: C 63.40, H 4.37, N 17.24\%. 6-(4-Methoxyanilino)(1,2,4-triazolo)(1,5-a)quinoline2-thiol 3f. Yield $71 \%$, m.p. $159{ }^{\circ} \mathrm{C}$. IR $(\mathrm{KBr}) \mathrm{v}_{\max }$ in $\mathrm{cm}^{-1}$ : $1284(\mathrm{~N}-\mathrm{N}), 1530(\mathrm{C}-\mathrm{C}$ of aromatic), $1618(\mathrm{C}=\mathrm{N})$, 3034 (aromatic $\mathrm{CH}), 3383(\mathrm{NH}) .{ }^{1} \mathrm{H}-\mathrm{NMR}\left(\mathrm{CDCl}_{3}\right)$ ठ: $3.85\left(\mathrm{~s}, 3 \mathrm{H}, \mathrm{OCH}_{3}\right), 5.70$ (bs, $\left.1 \mathrm{H}, \mathrm{NH}\right), 6.66-7.62(\mathrm{~m}$, 9H, Ar-H), 12.51 (s, 1H, HS). MS: m/z $322.09[\mathrm{M}]^{+}$. Elemental analysis $\left(\mathrm{C}_{17} \mathrm{H}_{14} \mathrm{~N}_{4} \mathrm{SO}\right)$, calcd: $\mathrm{C}$ 63.33, H 4.38, N 17.38\%, found: C 63.40, H 4.37, N 17.24\%. 6-(3-Aminoanilino)(1,2,4-triazolo)(1,5-a)quinoline-2thiol 3g. Yield $62 \%$, m.p. $121^{\circ} \mathrm{C}$. IR $(\mathrm{KBr}) \mathrm{v}_{\max }$ in $\mathrm{cm}^{-1}$ : $1280(\mathrm{~N}-\mathrm{N}), 1533(\mathrm{C}-\mathrm{C}$ of aromatic), $1621(\mathrm{C}=\mathrm{N})$, 3030 (aromatic $\mathrm{CH}), 3380(\mathrm{NH}) .{ }^{1} \mathrm{H}-\mathrm{NMR}\left(\mathrm{CDCl}_{3}\right)$ ঠ: 5.67 (bs, 1H, NH), 6.12 (bs, $\left.2 \mathrm{H}, \mathrm{NH}_{2}\right), 6.70-7.66(\mathrm{~m}$, 9H, Ar-H), 12.42 (s, 1H, HS). MS: m/z 307.09 [M] $]^{+}$. Elemental analysis $\left(\mathrm{C}_{16} \mathrm{H}_{13} \mathrm{~N}_{5} \mathrm{~S}\right)$, calcd: C 62.52, H 4.26, N 22.78\%, found: C 62.40, H 4.27, N 22.84\%. 6-(4-Aminoanilino)(1,2,4-triazolo)(1,5-a)quinoline-2thiol $3 \mathrm{~h}$. Yield $65 \%$, m.p. $130{ }^{\circ} \mathrm{C}$. IR $(\mathrm{KBr}) \mathrm{v}_{\max }$ in $\mathrm{cm}^{-1}$ : $1278(\mathrm{~N}-\mathrm{N}), 1531(\mathrm{C}-\mathrm{C}$ of aromatic), $1624(\mathrm{C}=\mathrm{N})$, 3032 (aromatic $\mathrm{CH}), 3377(\mathrm{NH}) .{ }^{1} \mathrm{H}-\mathrm{NMR}\left(\mathrm{CDCl}_{3}\right)$ ठ: 5.55 (bs, $1 \mathrm{H}, \mathrm{NH}), 6.17$ (bs, $\left.2 \mathrm{H}, \mathrm{NH}_{2}\right), 6.73-7.60(\mathrm{~m}$, 9H, Ar-H), 12.48 (s, 1H, HS). MS: m/z 307.09 [M] ${ }^{+}$. Elemental analysis $\left(\mathrm{C}_{16} \mathrm{H}_{13} \mathrm{~N}_{5} \mathrm{~S}\right)$, calcd: C 62.52, H 4.26, N 22.78\%, found: C 62.51, H 4.26, N 22.72\%.

\section{2-[(chloromethoxy)methylthio-N-substitutedphenyl] $-(1,2,4)$ triazolo(1,5-a)quinolin-6-amine 4a- $h$}

Compound 3a-h $(0.01 \mathrm{~mol}))$, potassium bicarbonate $(0.02 \mathrm{~mol})$, potassium iodide $(0.001 \mathrm{~mol})$ was taken in acetonitrile, stirred at room temperature for $30 \mathrm{~min}$, heated $70-80{ }^{\circ} \mathrm{C}$ for $2-3 \mathrm{~h}$. The progress of reaction was monitored by TLC for complete disappearance of $3 a-h$. On completion of reaction, mixture was diluted with water and extracted with ethyl acetate. The combined organic layer was washed with water, brine and then dried over anhydrous sodium sulphate. The dried organic layer was distilled under reduced pressure to furnish compounds $4 \mathrm{a}-\mathrm{h}$.

2-[(chloromethoxy)methylthio-N-phenyl]-(1,2,4)

triazolo(1,5-a)quinolin-6-amine 4a. Yield 68\%, m.p.135 ${ }^{\circ} \mathrm{C}$. IR (KBr) $\mathrm{v}_{\max }$ in $\mathrm{cm}^{-1}: 1280(\mathrm{~N}-\mathrm{N})$, $1527(\mathrm{C}-\mathrm{C}$ of aromatic), $1615(\mathrm{C}=\mathrm{N}), 3038$ (aromatic $\mathrm{CH}), 3380(\mathrm{NH})$. ${ }^{1} \mathrm{H}-\mathrm{NMR}\left(\mathrm{CDCl}_{3}\right) \delta: 3.00\left(\mathrm{~s}, 2 \mathrm{H}, \mathrm{CH}_{2}\right)$, 5.15 (s, 2H, $\mathrm{CH}_{2}-\mathrm{Cl}$ ), 5.80 (bs, $\left.1 \mathrm{H}, \mathrm{NH}\right), 6.95-7.90$ (m, $10 \mathrm{H}, \mathrm{Ar}-\mathrm{H})$. MS: m/z $370.07\left[\mathrm{M}^{+}\right.$. Elemental analysis 
$\left(\mathrm{C}_{18} \mathrm{H}_{15} \mathrm{~N}_{4} \mathrm{SClO}\right)$, calcd: C 58.30, H 4.08, N 15.11\%, found: C 58.26, H 4.07, N 15.27\%.

2-[(chloromethoxy)methylthio-N-(2-bromophenyl)]$(1,2,4)$ triazolo(1,5-a)quinolin-6-amine 4b. Yield 68\%, m.p.135 ${ }^{\circ} \mathrm{C}$. IR (KBr) $v_{\max }$ in $\mathrm{cm}^{-1}: 1280(\mathrm{~N}-\mathrm{N})$, 1527 (C-C of aromatic), $1615(\mathrm{C}=\mathrm{N}), 3038$ (aromatic $\mathrm{CH}), 3380(\mathrm{NH}) .{ }^{1} \mathrm{H}-\mathrm{NMR}\left(\mathrm{CDCl}_{3}\right) \delta: 3.10\left(\mathrm{~s}, 2 \mathrm{H}, \mathrm{CH}_{2}\right)$, $5.15\left(\mathrm{~s}, 2 \mathrm{H}, \mathrm{CH}_{2}-\mathrm{Cl}\right), 5.80$ (bs, $\left.1 \mathrm{H}, \mathrm{NH}\right), 6.95-7.90(\mathrm{~m}$, $9 \mathrm{H}, \mathrm{Ar}-\mathrm{H})$. MS: $\mathrm{m} / \mathrm{z} 449.97[\mathrm{M}]^{+}$. Elemental analysis $\left(\mathrm{C}_{18} \mathrm{H}_{14} \mathrm{~N}_{4} \mathrm{SBrClO}\right)$, calcd: C 48.07, $\mathrm{H} 3.14, \mathrm{~N} 12.46 \%$, found: C 48.12, H 3.12, N $12.44 \%$.

2-[(chloromethoxy)methylthio-N-(3-bromophenyl)]$(1,2,4)$ triazolo(1,5-a)quinolin-6-amine 4c. Yield $53 \%$, m.p.121 ${ }^{\circ} \mathrm{C}$. IR $(\mathrm{KBr}) \mathrm{v}_{\max }$ in $\mathrm{cm}^{-1}: 1274(\mathrm{~N}-\mathrm{N})$, 1520 (C-C of aromatic), $1626(\mathrm{C}=\mathrm{N}), 3028$ (aromatic $\mathrm{CH}), 3372(\mathrm{NH}) .{ }^{1} \mathrm{H}-\mathrm{NMR}\left(\mathrm{CDCl}_{3}\right) \delta: 3.08\left(\mathrm{~s}, 2 \mathrm{H}, \mathrm{CH}_{2}\right)$, $5.21\left(\mathrm{~s}, 2 \mathrm{H}, \mathrm{CH}_{2}-\mathrm{Cl}\right), 5.88$ (bs, $\left.1 \mathrm{H}, \mathrm{NH}\right), 6.79-7.85(\mathrm{~m}$, $9 \mathrm{H}, \mathrm{Ar}-\mathrm{H})$. MS: $\mathrm{m} / \mathrm{z} 449.97[\mathrm{M}]^{+}$. Elemental analysis $\left(\mathrm{C}_{18} \mathrm{H}_{14} \mathrm{~N}_{4} \mathrm{SBrClO}\right)$, calcd: C $48.07, \mathrm{H} 3.14, \mathrm{~N} 12.46 \%$, found: C 48.08, H 3.10, N $12.40 \%$.

2-[(chloromethoxy)methylthio-N-(4-bromophenyl)]$(1,2,4)$ triazolo(1,5-a)quinolin-6-amine 4d. Yield 57\%, m.p. $140{ }^{\circ} \mathrm{C}$. IR (KBr) $v_{\max }$ in $\mathrm{cm}^{-1}: 1277(\mathrm{~N}-\mathrm{N})$, $1524(\mathrm{C}-\mathrm{C}$ of aromatic), $1621(\mathrm{C}=\mathrm{N}), 3031$ (aromatic $\mathrm{CH}), 3372(\mathrm{NH}) .{ }^{1} \mathrm{H}-\mathrm{NMR}\left(\mathrm{CDCl}_{3}\right) \delta: 3.12\left(\mathrm{~s}, 2 \mathrm{H}, \mathrm{CH}_{2}\right)$, $5.21\left(\mathrm{~s}, 2 \mathrm{H}, \mathrm{CH}_{2}-\mathrm{Cl}\right), 5.87$ (bs, $\left.1 \mathrm{H}, \mathrm{NH}\right), 6.75-7.81(\mathrm{~m}$, $9 \mathrm{H}, \mathrm{Ar}-\mathrm{H}) . \mathrm{MS}: \mathrm{m} / \mathrm{z} 449.97[\mathrm{M}]^{+}$. Elemental analysis $\left(\mathrm{C}_{18} \mathrm{H}_{14} \mathrm{~N}_{4} \mathrm{SBrClO}\right)$, calcd: C 48.07, H 3.14, N 12.46\%, found: C 48.05, H 3.10, N $12.38 \%$.

2-[(chloromethoxy)methylthio-N-(3-methoxyphenyl)]$(1,2,4)$ triazolo(1,5-a)quinolin-6-amine 4e. Yield 56\%, m.p.179 ${ }^{\circ} \mathrm{C}$. IR $(\mathrm{KBr}) \mathrm{v}_{\max }$ in $\mathrm{cm}^{-1}: 1282(\mathrm{~N}-\mathrm{N})$, 1527 (C-C of aromatic), $1616(\mathrm{C}=\mathrm{N}), 3038$ (aromatic $\mathrm{CH}), 3375(\mathrm{NH}) .{ }^{1} \mathrm{H}-\mathrm{NMR}\left(\mathrm{CDCl}_{3}\right)$ ס: $3.15\left(\mathrm{~s}, 3 \mathrm{H}, \mathrm{OCH}_{3}\right)$, 3.71 (s, $2 \mathrm{H}, \mathrm{CH}_{2}$ ), 5.15 (s, 2H, CH $\mathrm{NH})$, 6.95-7.90 (m, 9H, Ar-H). MS: m/z $401.07\left[\mathrm{M}^{+}\right.$. Elemental analysis $\left(\mathrm{C}_{19} \mathrm{H}_{17} \mathrm{~N}_{4} \mathrm{SClO}_{2}\right)$, calcd: C 56.93, H 4.27, N 13.98\%, found: C 56.86, H 4.17, N $14.12 \%$.

2-[(chloromethoxy)methylthio-N-(4-methoxyphenyl)]$(1,2,4)$ triazolo(1,5-a)quinolin-6-amine 4f. Yield $51 \%$, m.p.188 ${ }^{\circ} \mathrm{C}$. IR $(\mathrm{KBr}) \quad \mathrm{v}_{\max }$ in $\mathrm{cm}^{-1}: 1278(\mathrm{~N}-\mathrm{N})$, 1521 (C-C of aromatic), $1620(\mathrm{C}=\mathrm{N}), 3035$ (aromatic $\mathrm{CH}), 3380(\mathrm{NH}) .{ }^{1} \mathrm{H}-\mathrm{NMR}\left(\mathrm{CDCl}_{3}\right) \delta: 3.23\left(\mathrm{~s}, 3 \mathrm{H}, \mathrm{OCH}_{3}\right)$, $3.68\left(\mathrm{~s}, 2 \mathrm{H}, \mathrm{CH}_{2}\right), 5.10$ (s, 2H, CH $\left.\mathrm{CH}_{2}-\mathrm{Cl}\right), 5.93$ (bs, $1 \mathrm{H}$, $\mathrm{NH}), 6.91-7.88(\mathrm{~m}, 9 \mathrm{H}, \mathrm{Ar}-\mathrm{H})$. MS: $\mathrm{m} / \mathrm{z} 401.07[\mathrm{M}]^{+}$. Elemental analysis $\left(\mathrm{C}_{19} \mathrm{H}_{17} \mathrm{~N}_{4} \mathrm{SClO}_{2}\right)$, calcd: $\mathrm{C}$ 56.93, H 4.27, N 13.98\%, found: C 56.90, H 4.20, N $14.01 \%$.

2-[(chloromethoxy)methylthio-N-(3-aminophenyl)]$(\mathbf{1}, 2,4)$ triazolo(1,5-a)quinolin-6-amine 4 g. Yield $59 \%$, m.p.116 ${ }^{\circ} \mathrm{C}$. IR $(\mathrm{KBr}) \mathrm{v}_{\max }$ in $\mathrm{cm}^{-1}: 1270(\mathrm{~N}-\mathrm{N})$, 1528 (C-C of aromatic), $1625(\mathrm{C}=\mathrm{N}), 3024$ (aromatic

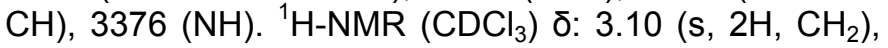
4.15 (bs, $2 \mathrm{H}, \mathrm{NH}_{2}$ ), 5.02 (s, 2H, CH $\mathrm{CH}_{2}-\mathrm{Cl}$ ), 5.87 (bs, $1 \mathrm{H}$, $\mathrm{NH})$, 6.75-7.81 (m, 9H, Ar-H). MS: m/z $385.08[\mathrm{M}]^{+}$.
Elemental analysis $\left(\mathrm{C}_{18} \mathrm{H}_{16} \mathrm{~N}_{5} \mathrm{SClO}\right)$, calcd: C $56.03, \mathrm{H}$ 4.18, N 18.15\%, found: C 56.11, H 4.14, N 18.28\%.

2-[(chloromethoxy)methylthio- $\mathrm{N}$-(4-aminophenyl)]$(1,2,4)$ triazolo(1,5-a)quinolin-6-amine $4 \mathrm{~h}$. Yield $49 \%$, m.p.145 ${ }^{\circ} \mathrm{C}$. IR (KBr) $v_{\max }$ in $\mathrm{cm}^{-1}: 1278(\mathrm{~N}-\mathrm{N})$, $1533(\mathrm{C}-\mathrm{C}$ of aromatic), $1628(\mathrm{C}=\mathrm{N}), 3028$ (aromatic $\mathrm{CH}), 3371(\mathrm{NH}) .{ }^{1} \mathrm{H}-\mathrm{NMR}\left(\mathrm{CDCl}_{3}\right)$ ס: $3.07\left(\mathrm{~s}, 2 \mathrm{H}, \mathrm{CH}_{2}\right)$, 4.12 (bs, $2 \mathrm{H}, \mathrm{NH}_{2}$ ), 5.10 (s, $2 \mathrm{H}, \mathrm{CH}_{2}-\mathrm{Cl}$ ), 5.99 (bs, $1 \mathrm{H}$, $\mathrm{NH})$, 6.64-7.80 (m, 9H, Ar-H). MS: m/z $385.08[\mathrm{M}]^{+}$. Elemental analysis $\left(\mathrm{C}_{18} \mathrm{H}_{16} \mathrm{~N}_{5} \mathrm{SClO}\right)$, calcd: $\mathrm{C}$ 56.03, H 4.18, N 18.15\%, found: C 56.00, H 4.20, N $18.20 \%$.

\section{PHARMACOLOGY}

Compounds 4a-h were evaluated for antimicrobial, insecticidal and antihelmintic activity (Table 1, 2, and 3).

\section{Antimicrobial Screening}

All the newly synthesized compounds $4 a-h$ were screened for their antibacterial and antifungal activity. All the bacterial as well as fungal strains were clinical isolates, identified with conventional morphological and biochemical methods. The microorganisms employed antibacterial studies were Staphylococcus aureus, Escherichia coli and Klebsiella pneumoniae. Disk diffusion method [39-40] was used for determination of the preliminary antibacterial activity. Disks measuring $6.25 \mathrm{~mm}$ in diameter were punched from Whatman no. 1 filter paper. Batches of 100 disks were dispensed to each screw-capped bottle and sterilized by dry heat at $140^{\circ} \mathrm{C}$ for an hour. The test compounds were prepared with different concentrations using DMF. One milliliter containing 100 times the amount of chemical in each disk was added to each bottle, which contained 100 disks. Disks of each concentration were for placed in triplicate in nutrient agar medium seeded with fresh bacteria separately. The incubation was carried out at $37{ }^{\circ} \mathrm{C}$ for $24 \mathrm{~h}$. Ampicillin trihydrate and fluconazole were used as standard drugs. Solvent and growth controls were kept and zones of inhibition were noted. The bacterial inhibition values $(\mathrm{mm})$ of the tested compounds against the tested bacterial strains are recorded in Table 1. On the other hand, the newly prepared quinoline compounds were screened for their in vitro antifungal activity against Aspergillus fumigatus (plant isolate), Candida albicans and Candida glabrata in DMF by the serial plate dilution method [41-42]. Sabouraud's agar media were prepared by dissolving peptone $(1 \mathrm{~g})$, D-glucose $(4 \mathrm{~g})$, and agar $(2 \mathrm{~g})$ in distilled water $(100 \mathrm{~mL})$ and adjusting the $\mathrm{pH}$ to 5.7 . Normal saline was used to make a suspension of the spore of fungal strain for lawning. A loopful of particular fungal strain was transferred to $3 \mathrm{~mL}$ saline to get a

Hament Panwar et al. 
Table-1. Antimicrobial screening of 2-[(chloromethoxy)methylthio-N substituted phenyl]-(1,2,4)triazolo(1,5-a)quinolin6-amine 4a-h.

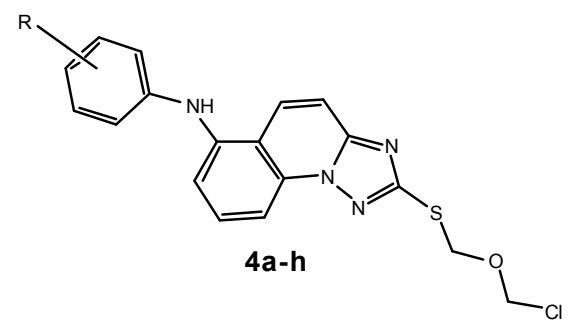

\begin{tabular}{|c|c|c|c|c|c|c|c|}
\hline \multirow[t]{2}{*}{ Compound } & \multicolumn{4}{|c|}{ Antibacterial activity (mm) } & \multicolumn{3}{|c|}{ Antifungal activity (mm) } \\
\hline & $\mathrm{R}$ & S. aureus & E. coli & K. pneumoniae & A. fumigatus & C. albicans & C. glabrata \\
\hline $4 a$. & $\mathrm{H}$ & - & - & - & 6 & - & - \\
\hline 4b. & $2-\mathrm{Br}$ & - & 6 & 5 & - & 6 & - \\
\hline $4 c$. & $3-\mathrm{Br}$ & - & - & 6 & 6 & - & 6 \\
\hline $4 \mathrm{~d}$. & $4-\mathrm{Br}$ & - & 6 & - & - & - & 8 \\
\hline $4 e$. & $3-\mathrm{OCH}_{3}$ & - & 5 & - & 8 & 6 & 10 \\
\hline $4 f$. & $4-\mathrm{OCH}_{3}$ & 6 & 6 & 10 & 14 & 10 & 8 \\
\hline $4 \mathrm{~g}$. & $3-\mathrm{NH}_{2}$ & - & 5 & - & 5 & - & 5 \\
\hline $4 \mathrm{~h}$. & $4-\mathrm{NH}_{2}$ & - & 5 & 6 & 6 & 6 & 6 \\
\hline $\begin{array}{l}\text { Ampicillin } \\
\text { trihydrate }\end{array}$ & & 16 & 16 & 20 & - & - & - \\
\hline Fluconazole & & - & - & - & 20 & 15 & 15 \\
\hline DMF (control) & & - & - & - & - & - & - \\
\hline
\end{tabular}

Table-2. Insecticidal activity of 2-[(chloromethoxy) methylthio-N-substitutedphenyl]-(1,2,4)triazolo(1,5-a)qui nolin-6-amine 4a-h at two different concentrations (KD value in $\min$.).

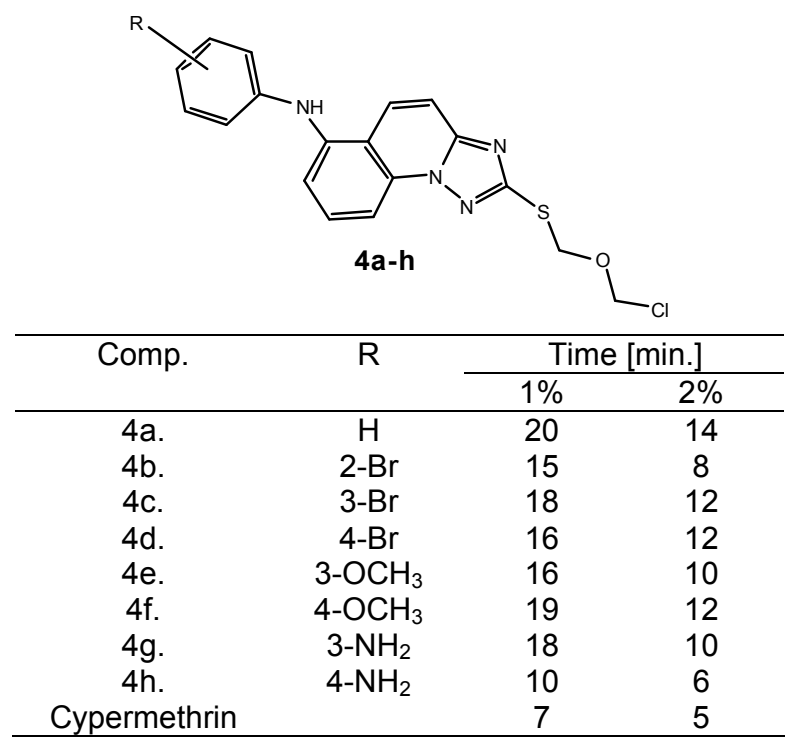

suspension of the corresponding species. Agar media $(20 \mathrm{~mL})$ was poured into each petri dish. Excess suspension was decanted and the plates were dried by placing in an incubator at $37^{\circ} \mathrm{C}$ for $1 \mathrm{~h}$. Using an agar punch wells were made into each well labeled. A control
Table-3. Anthelmintic activity of 2-[(chloromethoxy) methylthio-N-substitutedphenyl]-(1,2,4)triazolo(1,5-a) quinolin-6-amine 4a-h against Pheretima posthuma (paralytic and lethal time in min.).

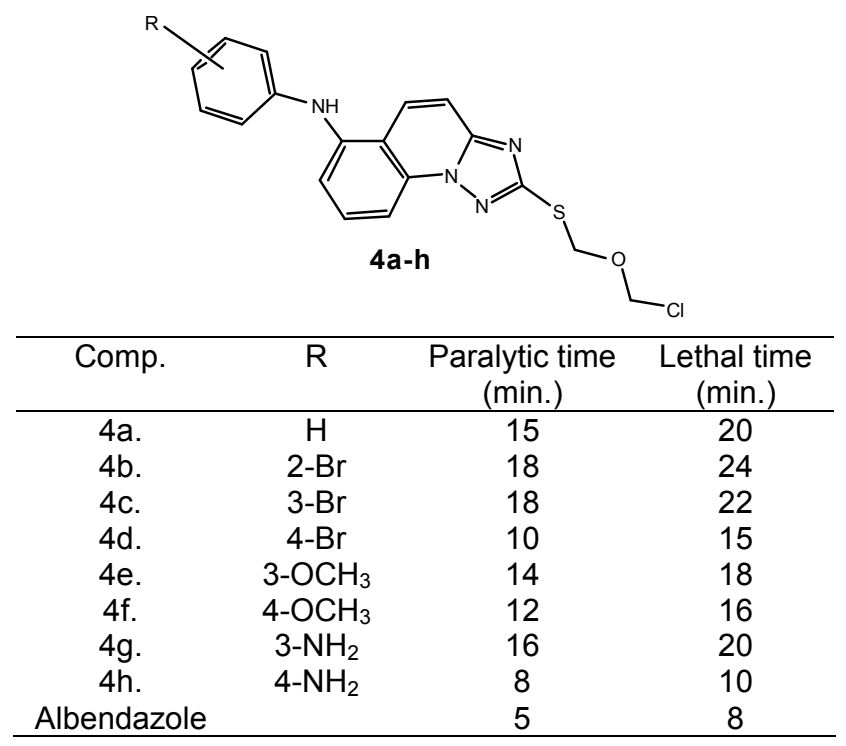

was also prepared in triplicate and maintained at $37^{\circ} \mathrm{C}$ for 3-4 days. Antifungal activity was determined by measuring the diameter of the inhibition zone. The fungicidal inhibitory $(\mathrm{mm})$ values of the tested 
compounds against the tested fungal species are recorded in Table 1.

\section{Insecticidal Study}

Periplaneta americana was taken for insecticidal study and 1 and $2 \%$ solutions of the derivatives $4 a-h$ were injected in the abdominal of $P$. americana with the help of micro syringe. The time of death had been noted as KD (Knock promising to moderate activity) value. Cypermethrin was used as standard drug. At the time of death the antennae of $P$. americana became motionless, the appendages shrunk and folded towards the ventral side and cockroach lay dorsally [43] (Table 2).

\section{Anthelmintic Activity}

Indian adult earthworms (Pheretima posthuma) were collected from moist soil and washed with normal saline to remove all faecal matter and used for the anthelmintic activity. All the synthesized derivatives $4 a-h$ were dissolved in minimum amount of DMF and the volume adjusted to $10 \mathrm{~mL}$ with saline water. All solutions of synthesized derivatives and drugs solutions were freshly prepared. Groups of six earthworms were released into desired formulations and the paralytic and lethal time noted. Albendazole was used as standard drug. Observations were made for the time taken for paralysis and death of individual worms. Paralysis was said to occur when the worms did not receive even in normal saline. Death was concluded when the worms lost their motility followed by fading away of their body color [44-47] (Table 3).

\section{RESULT AND DISCUSSION}

\section{Chemistry}

Synthesis was carried out by conventional methodology as sketched in scheme-1. Synthetic designing was started by the reaction of 5-bromo coumarin with thiosemicarbazide in presence of triethylamine (TEA) to obtain 1-(2-oxoquinolin-1(2H)yl)thiourea 1 which further on cyclised into 6-bromo(1,2,4-triazolo)(1,5-a) quinoline-2-thiol i.e. compound 2. The latter was anilated by the reaction of different substituted aromatic amines to afford 6-(substituted phenylamino)(1,2,4-triazolo)(1,5-a)quinoline-2-thiol; 3ah. Condensation reaction of compounds $3 a-h$ with bis(chloromethyl) ether produced target derivatives, 2[(chloro methoxy)methylthio-N-substitutedphenyl]-(1,2,4) triazolo(1,5-a)quinolin-6-amine; 4a-h.

\section{Biological evaluation}

The synthesized substituted quinolines 4a-h evaluated for antimicrobial, insecticidal and anthilmintic activity. Staphylococcus aureus. Escherichia coli, Klabsiella pneumoniae, Aspergillus fumigatus (plant isolate), Candida albicans, Candida glabrata pathogens were used for antimicrobial activity. Insecticidal activity performed against Periplaneta americana. Pheretima posthuma were used for the anthelmintic activity. All screened derivatives were found active against gram negative bacterial strains except derivative $4 \mathrm{f}$. Derivative $4 \mathrm{f}$ demonstrated inhibitory profile against all gram positive as well as gram negative pathogens. Quinoline derivative $4 \mathrm{f}$ and $4 \mathrm{~h}$ showed significant biological activity. Compound $4 a$ was found active against $A$. fumigatus while compound $4 \mathrm{~b}$ inhibited the growth of $E$. coli, $K$. pneumoniae and $C$. albicans. Derivative $4 \mathrm{c}$ showed no inhibition against $S$. aureus, $E$. coli and $C$. albicans. It was active against $K$. pneumoniae, A. fumigatus and C. glabrata. Compound $4 \mathrm{~d}$ asserted inhibition against $E$. coli and C. glabrata only. Compound $4 \mathrm{e}$ illustrated improved microbial inhibition. It inhibited the growth of $E$. coli, A. fumigatus and C. albicans, C. glabrata. Growths of all bacterial and fungal strains were inhibited by the compound $4 \mathrm{f}$ with good potency. No inhibitory action against $S$. aureus, $K$. pneumoniae and $C$. albicans was elicited by derivative $4 \mathrm{~g}$. Compound $4 \mathrm{~h}$ was active against all pathogens except $S$. aureus. It demonstrated poor antimicrobial array. Insecticidal activity results explored that among the tested compounds $4 \mathrm{a}-\mathrm{f}$, compound $4 \mathrm{~h}$ showed significant insecticidal potential in term of KD values with respect to used standards. Anthelmintic activity screening figures depicted mild to moderate potential of quinoline derivatives $4 a-h$. Paralytic and lethal time result summary elucidated the efficacy of derivative $4 \mathrm{~h}$ than the others tested derivatives.

\section{CONCLUSION}

Biological evaluation results revealed that 2$\{$ (chloromethoxy)methyl\}thio- $N$-(4-methoxyphenyl)[1,2,4] triazolo[1,5-a]quinolin-6-amine i.e. $4 f$ displayed significant biological activity. Screening data explored that 2-\{(chloromethoxy)methyl\}thio- $N$-(4-methoxyphenyl) $[1,2,4]$ triazolo[1,5-a]quinolin-6-amine showed broad antibacterial as well as antifungal spectrum in comparison to used standard drugs.

While, among the all tested compounds; compound 4 h i.e. $2-\{$ (chloromethoxy)methyl $\}$ thio- $N$-(4aminophenyl)[1,2,4]triazolo[1,5-a]quinolin-6-amine also showed moderate insecticidal as well as anthelmintic activity. On the basis of SAR, it is elicited that 2$\{$ (chloromethoxy)methyl\}thio- $N$-(4-methoxyphenyl) $[1,2,4]$ 
triazolo[1,5-a] quinolin-6-amine, bearing 4-methoxy substitution caused better efficacy against the all used pathogens while on the other hand derivative $4 \mathrm{~h}$ i.e. 2$\{$ (chloromethoxy)methyl\}thio- $N$-(4-aminophenyl) $[1,2,4]$ tria zolo[1,5-a]quinolin-6-amine bearing 4-amino group resulted into better insecticidal as well as anthelmintic activity. So 4-methoxy substitution and 4-amino enhance biological activity of the derived quinolines.

\section{ACKNOWLEDGEMENT}

We are thankful for SAIF, Punjab University, India for spectral, elemental analysis and Department of Pathology, L.L.R.M. Medical College, India for biological activities.

\section{REFERENCES}

1. Bruneto, J., 1999, Pharmacognosy, Phytochemistry, Medicinal Plants, $2^{\text {nd }}$ ed., Intercept Ltd., Hampshire, U. K., 263-277.

2. Mulwad, V.V., and Pawar, R.B., 2003, Indian J. Chem., 42B, 2091-2096.

3. Sharma, P., and Pritmani, S., 1999, Indian J. Chem., 38B, 1139-1142.

4. Emmanuel-Giota, A.A., Fylaktakidou, K.C., Hadjipavlou-Litina, D.J., Litinas, K.E., and Nicolaides, D.N., 2001, J. Heterocycl. Chem., 38, 3, 717-722.

5. Pratibha, S., and Shreeya, P., 1999, Indian J. Chem., 38, 1139-1142.

6. Rajanarendar, E., Karunakar, D., and Srinivas, M., 2004, Indian J. Chem., 43B, 643-648.

7. Anklekar, K.Y., Lakkannavar, C.D., Kulkarni, G.M., and Kulkarni, M.V., 2003, Indian J. Chem., 42B, 1548-1550.

8. Patonay, T., Litkei, G.Y., Bognar, R., Erdei, J., and Misztic, C., 1984, Pharmazie, 39, 86-91.

9. Shaker, R.M., 1996, Pharmazie, 51, 148-151.

10. El-Farargy, A.F., 1991, Egypt. J. Pharm. Sci., 32, 625-632.

11. Liu, B., Xie, L-G., Xu, X-H., and Li, Y-H., 2011, Chin. J. Org. Chem., 31, 12, 2067-2073.

12. Raev, L.D., Voinov, E., Ivanov, I.C., and Popov, D., 1990, Pharmazie, 45, 9, 696.

13. Nofal, Z.M., El-Zahar, M., and Abd El-Karim, S., 2000, Molecules, 5, 2, 99-113.

14. Xie, L., Tukeuchi, Y., Consetino, L.M., and Lee, K.H., 1999, J. Med. Chem., 42, 2662-2672.

15. Manolov, I., and Danchev, N.D., 1995, Eur. J. Med. Chem., 30, 6, 531-536.

16. Ragavendran, J.V., Sriram, D., Patel, S.K., Reddy, I.V., Bharathwajan, N., Stables, J., and Yogeeswari, P., 2007, Eur. J. Med. Chem., 42, 2, 146-151.
17. Rahman, V.P.M., Mukhtar, S., Ansari, W.H., and Lemiere, G., 2005, Eur. J. Med. Chem., 40, 2, 173-184.

18. Zora, M., and Gormen, M., 2007, J. Organomet. Chem., 692, 22, 5026-5032.

19. Ito, T., Fraser, I.P., Yeo, Y., Highley, C.B., Bellas, E., and Kohane, D.S., 2007, Biomaterials, 28, 10, 1778-1786.

20. Acharya, B.N., Saraswat, D., and Kaushik, M.P., 2008, Eur. J. Med. Chem., 43, 12, 2840-2852.

21. O'Kennedy, R., and Thornes, R.D., 1997, Coumarins-Biology, Applications and Mode of Action, John Wiley \& Sons Ltd., Chichester, Eds., 315.

22. Zahradnik, M., 1992, The Production and Application of Fluorescent Brightening Agent, John Wiley \& Sons Ltd., Chichester, England.

23. Schafer, F.P. (Eds.), 1990, Dye Lasers, $3^{\text {rd }}$ ed., Springer-Verlag, Berlin.

24. Duarte, F.J., and Hillman L.W. (Eds.), 1990, Dye Laser Principles, Academic, New York.

25. Duarte F.J., 2003, Tunable Laser Optics, ElsevierAcademic, New York, Appendix of Laser Dyes.

26. El Ashry, E.H., Kassem, A.A.K., Abdel-Hameed, H.M., Louis, F., Khattab, S.A.N., and Aouad, M.R., 2009, Carbohydr. Res., 344, 6, 725-733.

27. Cansız, A., Koparır, M., and Demirdag, A., 2004, Molecules, 9, 4, 204-212.

28. Liu, J., Li, L., Dai, H., Liu, Z., and Fang, J., 2006, J. Organomet. Chem., 691, 12, 2686-2690.

29. Ye, X-X., Chen, Z-F., Zang, A-J., and Zang, L-X., 2007, Molecules, 12, 6, 1202-1209.

30. Sztanke, M., Tuzimski, T., Rzymowska, J., Pasternak, K., and Kandefer-Szerszen, M., 2008, Eur. J. Med. Chem., 43, 2, 404-419.

31. Moise, M., Sunel, V., Profire, L., Popa, M., Debrieres, J., and Peptu, C., 2009, Molecules, 14, 7, 2621-2631.

32. Isloor, A.M., Kalluraya, B., and Shetty, P., 2009, Eur. J. Med. Chem., 44, 9, 3784-3787.

33. Farghaly, A., and El-Kashef, H., 2006, ARKIVOC, xi, 76-90.

34. El Ashry, E.S.H., Kassem, A.A., Abdel-Hamid, H., Khattab, S.A.N, and Aouad, M.R., 2006, ARKIVOC, xiv, 119-132.

35. Qin, X., Yu, H., Liu, J., Dai, B.G., Qin, Z., Zhang, X., Wang, T., and Fang, J., 2009, ARKIVOC, ii, 201-210.

36. Xu, W., Song, B., Bhadury, P., Song, Y., and Hu, D., 2010, Molecules, 15, 2, 766-779.

37. Khanmohammadi, H., Abnosi, M.H., Hosseinzadeh, A., and Erfantalab, M., 2008, Spectrochim. Acta, Part A, 71, 1474-1480.

38. Chai, B., Qian, X., Coa, S., Lui, H., and Song, G., 2003, ARKIVOC, ii, 141-145. 
39. Cruickshank, R., Duguid, P.J., Marion, P.B., and Swain, H.R., 1975, in Medicinal Microbiology, $12^{\text {th }}$ ed., Churchill Livingstone, London, U.K.

40. Collins, H.A., 1976, Microbiological Methods, $2^{\text {nd }}$ ed., Butterworth, London, U.K.

41. Khan, K.Z., 1997, In vitro and vivo screening techniques for bioactivity screening and evaluation, in Proceedings of the International Workshop on UNIDO-CDRI.

42. Varma, S.R., 1998, Antifungal Agents: Past, Present and Future Prospects, National Academy of Chemistry and Biology, Lucknow, India.

43. Ogino, K., Shimamura, H., Tomisawa, K., and Urushizaki, F., 1996, US Pat., 5, 484.
44. Nirmal, S.A., Malwadkar, G., and Laware, R.B., 2007, Songklanakarin J. Sci. Technol., 29, 3, 755-757.

45. Tambe, V.D., Nirmal, S.A., Jadhav, R.S., Ghogare, P.B., Bhalke, R.D., Girme, A.S., and Bhamber, R.S., 2006, Indian J. Nat. Prod., 22, 27-29.

46. Thorn, G.W., Adams, R.D., and Petersdrof, R.G., 1977, Harrison's Principles of Internal Medicine, McGraw Hill Co, New Zoology Department, Prof. M. Bhide, Department of York, 1088.

47. Vilgar, Z., 1984, Atlas of Medical Parasitology, PG Publishing kind assistance for performing antimicrobial studies House, Singapore, 216. 